\title{
Cartilage Thickness Measurement in the Sub-millimeter Range
}

\author{
Geert J. Streekstra ${ }^{1}$, Pieter Brascamp ${ }^{1}$, Christiaan van der Leij ${ }^{2}$, \\ René ter Wee ${ }^{1}$, Simon D. Strackee ${ }^{3}$, Mario Maas ${ }^{2}$, and Henk W. Venema ${ }^{1}$ \\ 1 Dept. Medical Physics, \\ g.j.streekstra@amc.uva.nl \\ 2 Dept. Radiology, \\ 3 Dept. Plastic and Reconstructive Surgery, \\ Academic Medical Center, Meibergdreef 9, 1105 AZ, Amsterdam, The Netherlands
}

\begin{abstract}
We present a method to measure cartilage thickness from $\mathrm{CT}$ images in the sub millimeter range. Current methods based on zero crossings of second derivatives across the cartilage layers are known to be biased in the sub millimeter range due to the finite width of the point spread function (PSF) of the imaging system.

We developed a method for accurate thickness measurements of such small layers by taking into account the effect of the PSF. To this end the orientation of the cartilage layers is estimated using gradient vector information in the cartilage region. Subsequently, a model of the attenuation profile across the cartilage layer is convolved with a measured PSF to obtain an intensity profile that is fitted to the image data.

Results of thickness estimates from simulated image data reveal that our method is unbiased in contrast to the method based on second derivative zero crossings. We illustrate the usefulness of our method by comparing measurement on CT arthrography images with results obtained from high resolution anatomical sections that served as a reference.

We conclude that incorporation of the PSF in the measurement method allows for accurate cartilage thickness estimates even in the sub millimeter range.
\end{abstract}

\section{Introduction}

In orthopaedics and musculoskeletal radiology detection of cartilage thickness is of importance in both clinical practice and in research on biomechanics of joint structures 1/23. In clinical practise cartilage thickness estimates can be used to stage joint disease in a primary diagnosis and in evaluation of pharmacological or surgical procedures. Moreover, cartilage thickness is an essential parameter in biomechanical models that describe the kinematic behavior of joint structures [4].

A lot of effort has been put into detection of cartilage in the knee joint 1 . 2,56,7,8,9]. In the knee cartilage thickness ranges typically from 2 to $5 \mathrm{~mm}$. Usually, the order of magnitude of the voxels size in the MR images used for 
cartilage measurement is $0.3 \times 0.3 \times 2.0 \mathrm{~mm}$. In particular the slice thickness of $2 \mathrm{~mm}$ cause overestimation of cartilage thickness when the image plane cuts obliquely through the cartilage layer [26]. The relative inaccuracy caused by a slice thickness of several millimeters will increase with decreasing cartilage thickness.

Comparison of MR based methods for cartilage thickness measurements with estimates from anatomical sections show that roughly 10-50\% of the measurements have an inaccuracy of more than $0.5 \mathrm{~mm}$ [15]. These inaccuracies may be acceptable for measurements in the knee but are unacceptable when cartilage thickness is in the sub millimeter range like in most part of the wrist and in the ankle [10].

Since in the sub millimeter regime the size of the Point Spread Function (PSF) of the imaging system is also in the order of magnitude of the cartilage thickness the PSF should be taken into account when measuring cartilage thickness. In literature on image based thickness measurement in both CT and MRI it is generally acknowledged that the finite width of the PSF limits accuracy of measurement in thin sheet structures 11 12,1314. These methods utilize second derivative zero crossings for thickness measurement. The observed bias in the thickness estimates start to become significant in the sub millimeter regime even in high resolution CT protocols where the FWHM of the PSF is approximately $0.7 \mathrm{~mm}$.

In this paper we propose a measurement procedure that strongly reduces PSF induced bias by incorporating the PSF directly into the thickness estimation method. The performance of the method is evaluated by thickness measurements in simulated images and images of cadaver wrists. In the cadaver wrists cartilage thickness estimates from CT arthrography images are compared to estimates in high resolution anatomical sections as obtained with an imaging cryomicrotome [15].

\section{Cartilage Thickness Measurement Procedure}

\subsection{Cartilage Thickness Estimation}

In a joint the cartilage layers are usually situated on the outer part of both bones to facilitate motion between two individual bones. As a result two parallel cartilage layers are close to each other. We can model the profile of X-ray attenuation coefficients by a series of step functions along a line perpendicular to the cartilage layers as depicted in Fig.1.

The profile reflects the different attenuation coefficients for trabecular bone, cortical bone, cartilage and the contrast medium that fills the gap between the two cartilage layers in CT arthrography images [16 17. In a 2D image the cartilage layers appear as relatively dark line structures surrounded by the the cortical bone layers and the contrast agent that appear as bright lines (see Fig.2.).

In the image formation process the attenuation coefficient profile is blurred because of the limited resolution of the CT scanner. This means from a mathematical point of view that an intensity profile in the image across the cartilage 


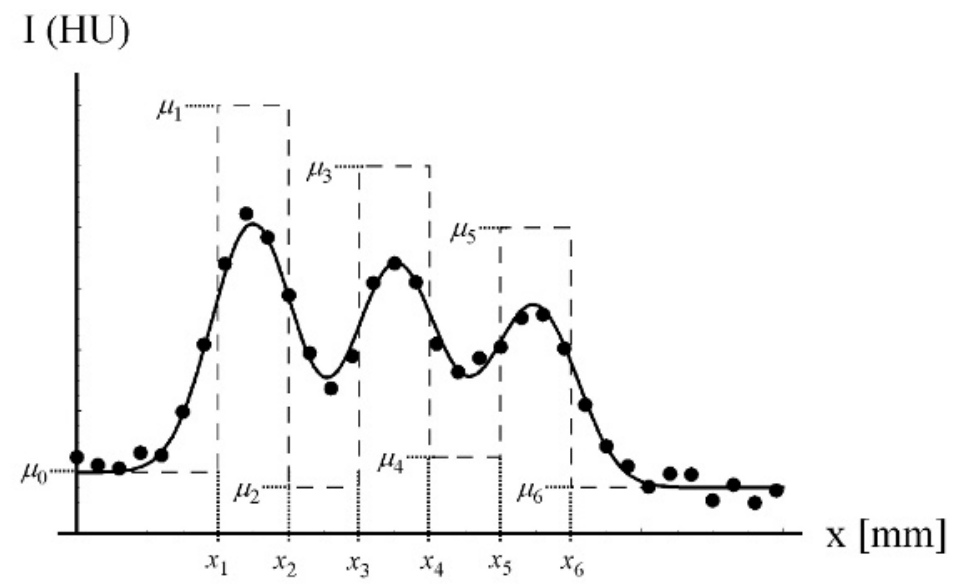

Fig. 1. Attenuation coefficient profile along a line perpendicular to the cartilage sheets (Dashed lines) and the convolution of the attenuation coefficient profile with the Point Spread Function (PSF) of the CT scanner (solid curve). The dots represent the noise corrupted discrete image points across the cartilage layers.

layers (Fig.1., solid curve.) is a 3D convolution of the attenuation coefficient profile (Fig.1., dashed lines.) with the PSF. Since in our case all relevant structures are approximately parallel with dimensions much larger than the size of the PSF the 3D convolution may be approximated by a 1D convolution along the attenuation coefficient profile. In the case that the PSF is isotropic and can be described by a 3D Gaussian [11] we find for the intensity profile across the cartilage layers:

$$
I(x)=\int_{-\infty}^{x_{1}} \mu_{0} g(x-\xi, \sigma) d \xi+\sum_{i=1}^{5} \int_{x_{i}}^{x_{i}+1} \mu_{i} g(x-\xi, \sigma) d \xi+\int_{x_{6}}^{\infty} \mu_{6} g(x-\xi, \sigma) d \xi
$$

In this equation $\mu_{i},(i=0 . .6)$ represent the attenuation coefficients of the different materials across the profile and $g(x, \sigma)$ is a one dimensional Gaussian with scale $\sigma$ :

$$
g(x, \sigma)=\frac{1}{\sqrt{2 \pi} \sigma} \exp \left(-\frac{1}{2} \frac{x^{2}}{\sigma^{2}}\right)
$$

Equation (1) shows that the intensity $I(x)$ at a certain $x$-position is influenced by the complete attenuation profile.

In the estimation of cartilage thickness the theoretical intensity profile as given in equation (1) is matched to a measured gray value profile in an image. 

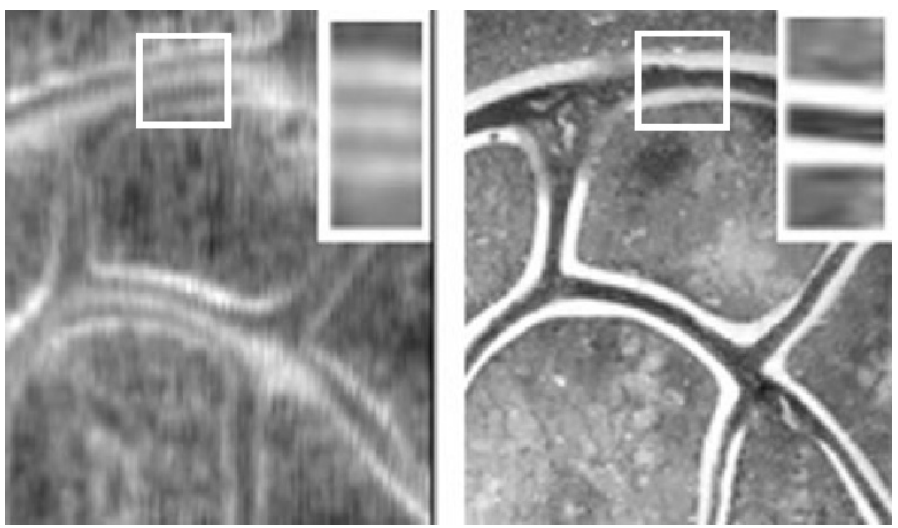

Fig. 2. Left: a slice of a 3D CT arthrography image showing the cartilage as dark lines between the bright cortical bone rims and contrast agent. Right: the corresponding anatomical section. The square regions are the positions of thickness estimates between radius and lunatum. The rectangular regions at the upper right corner of each image are magnifications of the ROI used for the thickness estimates.

For the matching a non-linear fit procedure is used to estimate the attenuation coefficients $\mu_{i}$ as well as the positions $x_{i}$ of the interfaces between different materials. The thicknesses $d_{1}$ and $d_{2}$ of the two adjacent cartilage layers are calculated from the positions of the estimated interface positions between cartilage-cortical bone and cartilage-contrast medium.

\subsection{Detection of Cartilage Sheet Orientation in 2D Image Slices}

In order to determine the thickness of the cartilage sheets from intensity profiles we first select a small region of interest (ROI) in a 2D image of the cartilage (Fig.2.) that contains the $3 \mathrm{D}$ vector normal to the cartilage sheet.

In order to obtain intensity profiles perpendicular to the cartilage the normal vector of the cartilage sheets in the $2 \mathrm{D}$ image is determined. This can be done by utilizing intensity gradients in the ROI. The transitions for one material to another are all in the same direction orthogonal to the direction of the cartilage sheets. Therefore by determining the average gradient, one can find the direction of the intensity profile from which the cartilage thickness is extracted.

The gradient components of the image in two orthogonal directions, $x$ and $y$, are calculated using Gaussian derivatives

$$
I_{x}=\frac{\partial g_{2}(x, y, \sigma)}{\partial x} \otimes I(x, y) \quad, \quad I_{y}=\frac{\partial g_{2}(x, y, \sigma)}{\partial y} \otimes I(x, y)
$$


where $\otimes$ is the $2 \mathrm{D}$ convolution operator and $g_{2}(x, y, \sigma)$ is the two-dimensional Gaussian

$$
g_{2}(x, y, \sigma)=\frac{1}{2 \pi \sigma^{2}} \exp \left(-\frac{x^{2}+y^{2}}{2 \sigma^{2}}\right)
$$

with $\sigma$ the scale of the Gaussian derivative kernel. By convolving the intensity with this Gaussian, the image is blurred for noise elimination while preserving derivative properties [18. For every pixel in the ROI, the angle of the intensity gradient vector with the $x$-axis is calculated using

$$
\theta=\arctan \left(\frac{I_{y}}{I_{x}}\right)
$$

To prevent the angles of positive and negative gradients in the same direction to cancel each other out, the intensity derivatives in both $x$ and $y$ directions are multiplied by -1 for all pixels with a negative derivative in the $y$ direction. Now a weighted average $\Theta$ of all angles with weight based on the magnitude of the gradients is made:

$$
\Theta=\frac{\sum_{i=1}^{n} \sum_{j=1}^{m}\left[\sqrt{I_{x}(i, j)^{2}+I_{y}(i, j)^{2}} \theta(i, j)\right]}{\sum_{i=1}^{n} \sum_{j=1}^{m} \sqrt{I_{x}(i, j)^{2}+I_{y}(i, j)^{2}}}
$$

where $n$ and $m$ are the width and height of the ROI, respectively. This is done because the largest gradients in the image are those on the edges of structures. Other (smaller) gradients are the result of noise. The influence of this noise is suppressed by taking a weighted average.

The local cartilage thickness is estimated by averaging the results of the fits of $n$ lines in the the ROI with dimensions $n \times m$. To estimate the error produced in this method, the bootstrap method [19] is used.

\section{$3 \quad$ Experiments}

\subsection{Simulations}

To gain insight into the potentials of the method we estimated the thickness of simulated 1D cartilage profiles. We compared our method with the commonly used method based on detection of second derivative zero crossings across the simulated cartilage layers 11,12,13 14.

The simulated profiles were constructed by convolving an attenuation profile of step functions with a Gaussian PSF (Eqs. (1) and (2)). The scale parameter $\sigma$ of the PSF was chosen to be $0.25 \mathrm{~mm}$ which is a value obtained in the highest resolution mode of current CT scanners. The voxel size was set to $0.2 \mathrm{~mm}$. We added random Gaussian noise to the simulated data with a SNR of 40 .

Initially all distances between steps in the attenuation profile (i.e. distances between $x_{i}$ and $x_{i+1}$, see Fig. 1.) were set to $1 \mathrm{~mm}$. Subsequently, the thickness of one of the cartilage layers $\left(d=x_{3}-x_{2}\right)$ was varied between 0.5 and $1.5 \mathrm{~mm}$. The signal to noise ration was set to 40 . 


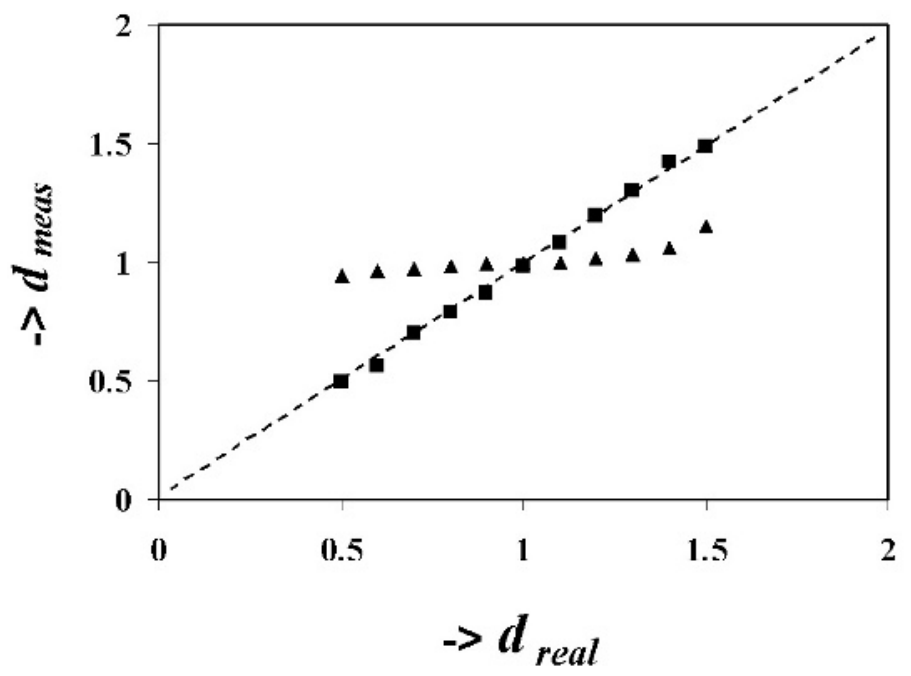

Fig. 3. Simulation based relationship between the real thickness of a cartilage layer $\left(d_{\text {real }}\right)$ and the estimated thickness $\left(d_{\text {meas }}\right)$. The triangles show the results of thickness estimated based on second derivative zero crossings across the cartilage layers. The squares show the results as obtained with our method that takes the PSF into account.

The results show that the method based on second derivative zero crossings show considerable bias in the estimated thickness (Fig. 3.). In comparison, thickness estimates with the present method shows virtually no bias. Only random variations appear to be present due to the small amount of noise added to the simulated intensity values.

\subsection{Comparison of Thickness Estimation in CT and Anatomical Sections}

To get an impression of the usefulness of our method in practise we compared thickness measurement from CT arthrography images with thickness estimates in high resolution anatomical sections as obtained with an imaging cryomicrotome 15] that served as a reference. The cryomicrotome images can be considered as an accurate reference since the width of the PSF of the cryomicrotome is approximately 20 times smaller than that of the CT-scanner (Mx8000, Philips) in the high resolution mode. The measured $\sigma$ of the isotropic Gaussian PSF of the CT-scanner is $0.255 \mathrm{~mm}$. The voxel size was set to $0.2 \mathrm{x} 0.2 \mathrm{x} 0.2 \mathrm{~mm}^{3}$.

The cartilage thickness was estimated from layers situated between the radius and the ulna (See indicated square regions in Fig. 2.). The thickness was measured at 10 different positions along the cartilage where the two layers are parallel. 


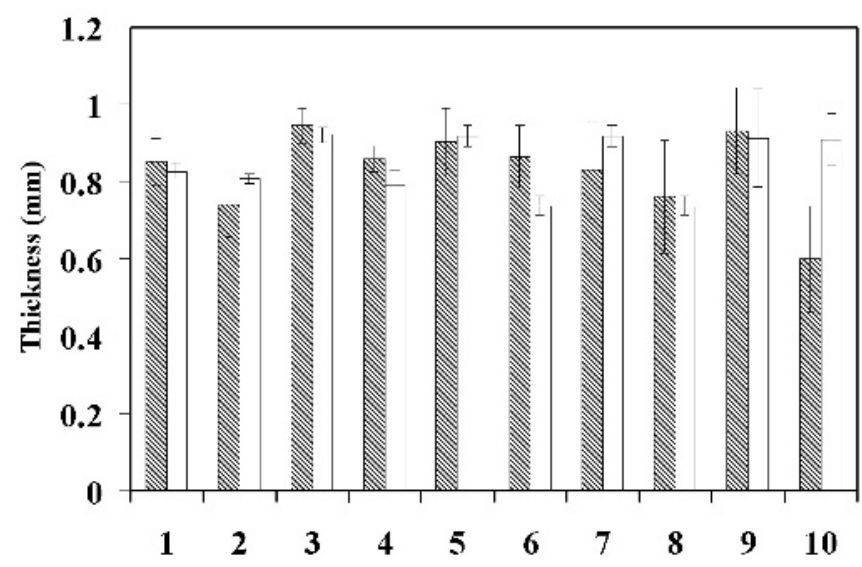

Fig. 4. Comparison of cartilage thickness estimated from CT arthography images (dashed bars) and from an anatomical section (open bars). The cartilage thickness was estimated from layers situated between the radius and the ulna (square regions in Fig. 2.)

As can be deduced from Fig. 4. the thickness estimated from the CT arthrography images correspond closely to those estimated from the cryomicrotome images in all cases except one.

\section{Discussion}

Measurement of thickness in sheet structures based on second derivative zero crossings is an accurate method if the size of the point spread function (PSF) of the imaging system is small compared to sheet thickness. In sheet structures below $1 \mathrm{~mm}$, like cartilage sheets in the wrist joint or the ankle, the width of the PSF is in the same order of magnitude as the cartilage thickness. In that case second derivative methods show considerable bias as shown in Fig. 3.

We presented a method that yields a bias free thickness estimator of parallel cartilage layers with a attenuation profile as shown in Fig. 1. The agreement of thickness estimates from CT images with estimates from anatomical sections is most promising. Residual differences may be due to poor SNR of the CT images as well as slight differences in the the exact locations in CT and cryomicrotome images. For a definitive validation of the method special attention should be paid to these aspects. 


\section{References}

1. Eckstein F., Sittek H., Gavazzeni A., Schulte E., Milz S., Kiefer B., Reiser M., Putz R. Magnetic resonance chondro-crassometry (MR CCM): A method for accurate determination of articular cartilage thickness. Magnetic Resonance in Medicine 35 (1), 89-96, 1996.

2. Losch A., Eckstein F., Haubner M., Englmeier K.H. A non-invasive technique for 3-dimensional assessment of articular cartilage thickness based on MRI Part 1: Development of a computational method. Magnetic Resonance Imaging 15 (7), 795-804, 1997.

3. Graichen H., Jakob J., von Eisenhart-Rothe R., Englmeier K.H., Reiser M., Eckstein F. Validation of cartilage volume and thickness measurements in the human shoulder with quantitative magnetic resonance imaging. Osteoarthritis and Cartilage 11(7), 475-482, 2003.

4. Blankevoort L., Kuiper J.H., Huiskes R., Grootenboer H.J. Articular Contact in a 3-Dimensional Model of the Knee. Journal of Biomechanics24 (11), 1019-1031, 1991.

5. Eckstein F., Gavazzeni A., Sittek H., Haubner M., Losch A., Milz S., Englmeier K.H., Schulte E., Putz R., Reiser M. Determination of knee joint cartilage thickness using three-dimensional magnetic resonance chondro-crassometry (3D MRCCM). Magnetic Resonance in Medicine 36 (2), 256-265, 1996.

6. Haubner M., Eckstein F., Schnier M., Losch A., Sittek H., Becker C., Kolem H., Reiser M., Englmeier K.H. A non-invasive technique for 3-dimensional assessment of articular cartilage thickness based on MRI Part 2: Validation using CT arthrography. Magnetic Resonance Imaging 15 (7), 805-813, 1997.

7. Stammberger T., Eckstein F., Englmeier K.H., Reiser M. Determination of 3D cartilage thickness data from MR imaging: Computational method and reproducibility in the living. Magnetic Resonance in Medicine 41(3), 529-536, 1999.

8. Stammberger T., Eckstein F., Michaelis M., Englmeier K.H., Reiser M. Interobserver reproducibility of quantitative cartilage measurements: Comparison of B-spline snakes and manual segmentation. Magnetic Resonance Imaging 17 (7), 1033-1042, 1999.

9. Glaser C., Faber S., Eckstein F., Fischer H., Springer V., Heudorfer L., Stammberger T., Englmeier K.H., Reiser M. Optimization and validation of a rapid high-resolution T1-w 3D FLASH water excitation MRI sequence for the quantitative assessment of articular cartilage volume and thickness. Magnetic Resonance Imaging 19 (2), 177-185, 2001.

10. Shepherd D.E.T., Seedhom B.B. Thickness of human articular cartilage in joints of the lower limb. Annals of the Rheumatic Diseases 58 (1), 27-34, 1999.

11. Prevrhal S., Engelke K., Kalender W.A. Accuracy Limits for the Determination of Cortical Width and Density: The Influence of Object Size and CT Imaging Parameters. Physics in Medicine and Biology 44 (3), 751-764, 1999.

12. Prevrhal S., Fox J.C., Shepherd J.A., Genant H.K. Accuracy Of CT-Based Thickness Measurement of thin Structures: Modeling of Limited Spatial Resolution in all three Dimensions. Medical Physics 30 (1), 1-8, 2003.

13. Sato, Y., Nakanishi, K., Tanaka, H., Nishii, T., Sugano, N., Nakamura, H., Ochi, T., Tamura, S. Limits to the accuracy of 3D thickness measurement in magnetic resonance images. Lecture Notes in Computer Science 2208, 803-810, 2001. 
14. Sato Y., Tanaka H., Nishii T., Nakanishi K., Sugano N., Kubota T., Nakamura H., Yoshikawa H., Ochi T., Tamura S. Limits on the accuracy of 3-D thickness measurement in magnetic resonance images - Effects of voxel anisotropy. IEEE Transactions on Medical Imaging 22 (9), 1076-1088, 2003.

15. Kelly J.J., Ewen J.R., Bernard S.L., Glenny R.W., Barlow C.H. Regional blood flow measurements from fluorescent microsphere images using an Imaging CryoMicrotome. Review of Scientific Instruments 71 (1), 228-234, 2000.

16. Boven F., Bellemanns M.A., Geurts J., Potvliege R. A comparative study of the patellofemoral joint on axial roentgenogram axial arthrogram and computed tomography following arthrography. Skeletal Radiology 8, 179-181, 1982.

17. Ihara H. Double contrast CT arthrography of the cartilage of the patellofemoral joint. Clinical Orthopeadics 198, 50-55, 1985.

18. Ter Haar Romeny B.M. Front-End Vision and Multi-Scale Image Analysis. Kluwer Academic Publisher, Dordrecht 2003, p 102.

19. Press W.H., Teukolsky S.A., Vetterling W.T., Flannery, B.P. Numerical Recipes in C. Cambridge University Press, New York 1992, p. 691. 